\title{
Influence of Expendable Current Profiler Probe on Induced Electric Field of Ocean Currents
}

\author{
Qisheng Zhang, Xiao Zhao, Xinyue Zhang, Jianen Jing, Shenghui Liu, \\ Shuhan Li, and Zhenzhong Yuan
}

China University of Geosciences, Beijing 100083, China

Correspondence should be addressed to Xiao Zhao; zhaoxiao@cugb.edu.cn

Received 6 June 2016; Accepted 23 August 2016

Academic Editor: Nazrul Islam

Copyright (C) 2016 Qisheng Zhang et al. This is an open access article distributed under the Creative Commons Attribution License, which permits unrestricted use, distribution, and reproduction in any medium, provided the original work is properly cited.

\begin{abstract}
The expendable current profiler (XCP) is a new instrument that is internationally used to rapidly monitor ocean currents in marine environments. The most crucial part of this instrument is the XCP probe. Since the probe is of high electrical resistance, it acts almost like an insulator with respect to seawater. Placing it into the induced electric field (IEF) of seawater therefore yields a certain level of influence over the electric field. Therefore, in order to improve the accuracy of XCP measurements, the conditions associated with this influence can be used to guide the design of XCP probes; at the same time, these can also serve as reference points in order to provide technical support for the processing of XCP data on ocean currents. To this end, computer-based numerical simulations and laboratory-based physical simulations are used in this study. The results showed that after an XCP probe (diameter: $5 \mathrm{~cm}$; length: $52 \mathrm{~cm}$ ) was inserted into seawater, the voltage difference of ocean currents at both ends of the electric field sensor placed above the XCP probe increased by a factor of 1.85 (as compared to the case in which there is no influence from the probe).
\end{abstract}

\section{Introduction}

The United States began researching expendable temperature and velocity profilers (XTVPs) as early as the 1970s and in 1978 successfully developed the first XTVP $[1,2]$. Between 1979 and 1980, a research team led by Sanford and Sippican company made coproduction and sea trial of several hundred XTVP probes and obtained preliminary detection results $[3,4]$. The company subsequently changed the name of this device from "XTVP" to "expendable current profiler" (XCP) and launched production of the instrument, which became widely used in marine surveys, scientific research, and national defense $[4,5]$. The XCP is a type of expendable profiling instrument for marine environments and can obtain the profile information of ocean currents rapidly [6]. For the first time in China, we have conducted an in-depth study of various XCP technologies [7, 8]. After independent research and development, China's first set of XCP equipment was manufactured using precision design [7]. Multiple marine tests indicated that placing the XCP probe into seawater changed the IEF of ocean currents. The XCP can be deployed through a probe launch or be manually cast from the carrying platform of ships, submarines, and aircraft. It can quickly measure ocean currents and temperature profiles while sinking and can calculate water depth based on the probe's sinking velocity $[9,10]$. Data are then transferred to the carrying platform through wired or wireless communication modes, and the real-time data of ocean currents and temperature variation with respect to water depth is obtained after data processing. To verify the accuracy of the XCP measurements, an acoustic Doppler current profiler (ADCP) is used for comparison. The used ADCP is an OS-75K from the RDI Company. The results of seawater experiments showed that the ocean current velocities measured by XCP and ADCP are very similar [7].

We found that placing this instrument in seawater led to changes in the induced electric field (IEF) that is generated by the movement of seawater. Hence, in-depth studies on the influence of XCP probes on the IEF of ocean currents are required in order to improve detection accuracy [11, 12]. In this study, computer-based numerical simulations and laboratory-based physical simulations were used to examine the influence of the XCP probe on the IEF of ocean currents and to obtain the corresponding coefficients of influence. 


\section{Materials and Methods}

2.1. Integral Equation Method. The ellipsoidal probe was placed in homogeneous seawater with an electrical conductivity of $\sigma_{1}$. The probe itself has an electrical conductivity of $\sigma_{2}(\vec{r})$, which is a function of $r . \vec{r}$ represents the radius vector. In addition, because the influence of magnetic permeability is usually minimal compared to that of electrical conductivity, it was assumed that $\mu=\mu_{0}$.

It was assumed that the electric dipole source $J$ was located somewhere in space and that the time-harmonic factor was $e^{j \omega t}$. We began with Maxwell's equations for the frequency domain as follows:

$$
\begin{aligned}
\nabla \times E+i \omega \mu_{0} H & =0, \\
\nabla \times H-\sigma E & =J .
\end{aligned}
$$

The response of homogeneous seawater was defined as the primary field and was represented by the subscript " $b$ "; the primary field also satisfied the following Maxwell equations:

$$
\begin{aligned}
\nabla \times E_{b}+i \omega \mu_{0} H_{b} & =0, \\
\nabla \times H_{b}-\sigma_{1} E_{b} & =J .
\end{aligned}
$$

At this stage, the following equations were obtained by subtracting the formulas in (2) from the corresponding formulas in (1):

$$
\begin{array}{r}
\nabla \times\left(E-E_{b}\right)+i \omega \mu_{0}\left(H-H_{b}\right)=0, \\
\nabla \times\left(H-H_{b}\right)-\sigma E+\sigma_{1} E_{b}=0,
\end{array}
$$

where $\sigma$ is the practical conductivity value of the model. The conductivity values inside and outside of the probe equal $\sigma_{2}(\vec{r})$ and $\sigma_{1}$, respectively.

At this stage, (4) can be rewritten as follows:

$$
\nabla \times\left(H-H_{b}\right)-\sigma_{1}\left(E-E_{b}\right)+\left(\sigma_{1}-\sigma\right) E=0 .
$$

If the difference between the total and primary field is considered to be the secondary field (represented by the subscript " $a$ "), then (5) can be simplified as follows:

$$
\nabla \times H_{a}-\sigma_{1} E_{a}=J_{s},
$$

where the following equation holds:

$$
J_{s}=\left[\sigma_{2}(r)-\sigma_{1}\right] E .
$$

This is known as the scattered current, which would exist only within the probe.

Equation (3) can be similarly simplified:

$$
\nabla \times E_{a}+i \omega \mu_{0} H_{a}=0 .
$$

The secondary field can be treated as being caused by the scattered current $J_{e}$. Because $E_{e}=-\widehat{z} A+(1 / \widehat{y}) \nabla(\nabla \cdot A)$, the secondary field in the seawater can be calculated using the following equation:

$$
E_{a}=-i \omega \mu_{0} A_{a}-\nabla V_{a}
$$

where $A_{a}$ and $V_{a}$ are the secondary vector potential and secondary scalar under Lorentz condition, respectively:

$$
\begin{aligned}
& A_{a}(r)=\int_{v} J_{s}\left(r^{\prime}\right) g\left(r, r^{\prime}\right) d v^{\prime}, \\
& V_{a}(r)=-\frac{1}{\sigma_{1}} \int_{v} \nabla \cdot J_{s}\left(r^{\prime}\right) g\left(r, r^{\prime}\right) d v^{\prime} .
\end{aligned}
$$

In (10), $g\left(r, r^{\prime}\right)$ is Green's function for the scalar quantity of total space, which was derived from the following equation:

$$
g\left(r, r^{\prime}\right)=\frac{e^{-i k R}}{4 \pi R}
$$

where $R=\left|r=r^{\prime}\right|$ and $k^{2}=-i \omega \mu_{0} \sigma_{1}$.

When the probe is in half-space, an additional item must be added to (9) to reflect the influence of the interface. This additional item has been described by Hohman and Wannamaker et al. under the conditions of homogeneous and layered ground, respectively [13-15]. This leads to the following expression for the secondary field:

$$
E_{a}(r)=\int_{v} G_{E}\left(r, r^{\prime}\right) \cdot\left[\sigma_{2}(r)-\sigma_{1}\right] \cdot E\left(r^{\prime}\right) d v^{\prime} .
$$

As described previously, the electromagnetic field is made up of two portions, the primary and secondary fields:

$$
E(r)=E_{b}(r)+E_{a}(r) .
$$

Substituting (12), which represents the secondary field, into (13) yields the total electric field's singular Fredholm integral equation of the second kind:

$$
\begin{aligned}
E(r)= & E_{b}(r) \\
& +\int_{v} G_{E}\left(r, r^{\prime}\right) \cdot\left[\sigma_{2}(r)-\sigma_{1}\right] \cdot E\left(r^{\prime}\right) d v^{\prime},
\end{aligned}
$$

where $G_{E}\left(r, r^{\prime}\right)$ represents dyadic Green's function. This function, which was required because the direction of the electric field at location $r$ would be different from that of the source current at location $r^{\prime}$, can be derived using the following equation:

$$
G_{E}\left(r, r^{\prime}\right)=\frac{1}{\sigma_{1}}\left[k^{2} I-\nabla \nabla^{\prime}\right] g\left(r, r^{\prime}\right),
$$

where $\nabla^{\prime}$ represents the derivative for the prime coordinate system and $I$ is the unit dyadic.

The probe was partitioned into $N$ cubic units, each of which had a length of $\Delta[16]$. The electric conductivity within each unit was constant. Because the scattered current $J_{s}$ within each unit would also be constant, the integral equation (3) can be approximated as follows:

$$
E(r)=E_{b}(r)+\sum_{n=1}^{N} \int_{v n} G_{E}\left(r, r^{\prime}\right) d v^{\prime} \cdot\left[\sigma_{2 n}-\sigma_{1}\right] E^{n},
$$

where $\sigma_{2 n}$ and $E^{n}$ represent the electric conductivity and field of the $n$th unit, respectively. 
When $\Gamma\left(r, r^{\prime}\right)$ is used to represent the integral term of (16), the latter can be written as follows:

$$
E(r)=E_{b}(r)+\sum_{n=1}^{N} \Gamma\left(r, r^{\prime}\right) \cdot\left(\sigma_{2 n}-\sigma_{1}\right) E^{n},
$$

where $\Gamma$ is dyadic Green's function for a small current integration. It is different from $G$, which is dyadic Green's function for a relatively infinitesimal current element.

$$
\Gamma\left(r, r^{\prime}\right)=\int_{v n} G_{E}\left(r, r^{\prime}\right) d v^{\prime} .
$$

Under these conditions, the electric field at the centre of the $m$ th unit can be written as follows:

$$
E^{m}=E_{b}^{m}+\sum_{n=1}^{N} \Gamma_{m n} \cdot\left(\sigma_{2 n}-\sigma_{1}\right) E^{n} .
$$

After transposition, it can be written as

$$
\sum_{n=1}^{N}\left[\delta_{m n}-\left(\sigma_{2 n}-\sigma_{1}\right) \Gamma_{m n}\right] \cdot E^{n}=E_{b}^{m},
$$

where the following holds:

$$
\delta_{m n}= \begin{cases}I, & m=n \\ 0, & m \neq n .\end{cases}
$$

Here, $I$ is a $3 \times 3$ unit matrix, and 0 is the zero tensor.

When every $m$ is expressed using (20), the following block matrix equation can be obtained:

$$
[M][E]=-\left[E_{b}\right] ;
$$

in the matrix $[M]$, each element is itself a $3 \times 3$ matrix:

$$
M_{m n}=\left(\sigma_{2 n}-\sigma_{1}\right) \Gamma_{m n}-\delta_{m n}
$$

Equation (22) can then be used to solve for the value of the electric field at the centre of each unit within the probe. At this stage, the electric field at any position outside the probe can be obtained using (16).

\section{Results and Discussion}

\subsection{Numerical Simulations of the Probe's Influence on Marine IEFs}

3.1.1. Theoretical Model. A theoretical model for the XCP probe (Figure 1(a)) was first established for conducting numerical simulations [17]. In the figure, $A B$ and $A^{\prime} B^{\prime}$ refer to the electric dipoles. The used current was $1000 \mathrm{~A}$, and the used frequencies were $0,0.0001$, and $1 \mathrm{~Hz}$. The XCP probe was placed in an infinite amount of seawater with a resistivity of $0.33 \Omega \cdot \mathrm{m}$. A Cartesian coordinate system was then established by assuming that seawater flow only occurs at the sea surface. The origin is located at the sea surface, while the $y$-axis points in the direction of the ocean currents, and there are no ocean currents along the $x$-axis. The $x$-and $y$-axes were both

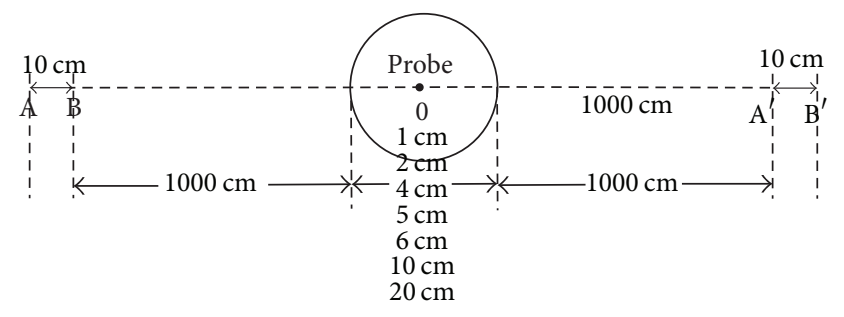

(a)

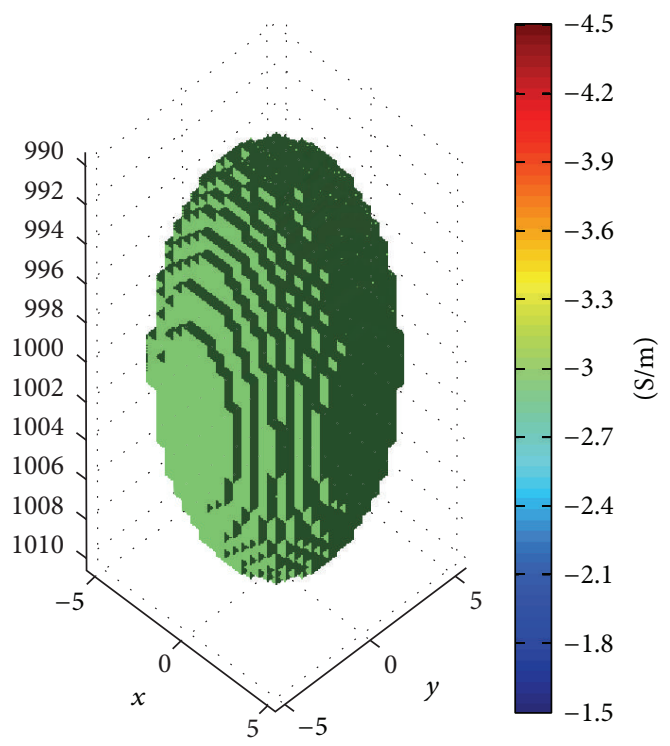

(b)

FIGURE 1: (a) Schematic diagram of the expendable current profiler (XCP) probe. (b) Mesh decomposition results for the probe model.

located at the sea surface, while the $z$-axis pointed upward and was perpendicular to the sea surface. The $x$-, $y$-, and $z$-axes conform to the right-hand rule. The conductivity of the probe was assumed to be $0 \mathrm{~S} / \mathrm{m}$, while the observation plane was located at $z=-1000 \mathrm{~cm}$. The length of the probe's minor axis was, respectively, at $1,2,4,5,6,10$, and $20 \mathrm{~cm}$, while that of its major axis along the $z$-direction was $52 \mathrm{~cm}$. Mesh decomposition was carried out for the probe model [18], resulting in $40 \times 40 \times 52$ grids (Figure 1(b)).

3.1.2. Probe's Influence on Electric Field Distribution. The integral equation method $[13,14]$ was used to carry out theoretical calculations of the probe model's electromagnetic response. Figures 2(a) and 2(b) show the distribution characteristics of the $E_{y}$ component of the disturbance field caused by the XCP probe along the $x$-and $y$-axis-label on the observation plane [19]. Along the direction of the $y$-axis, the electrical current field was affected by the high-resistance probe, causing a repulsion of the current or electric field. As can be seen from the figures, the electric field decreases as the observation points moved nearer to the probe along the $y$-axis, which exhibits negative anomalous characteristics. When the repulsed current line passes through the left and right sides of the XCP probe, the current density correspondingly increases. This results in positive anomalous characteristics. 

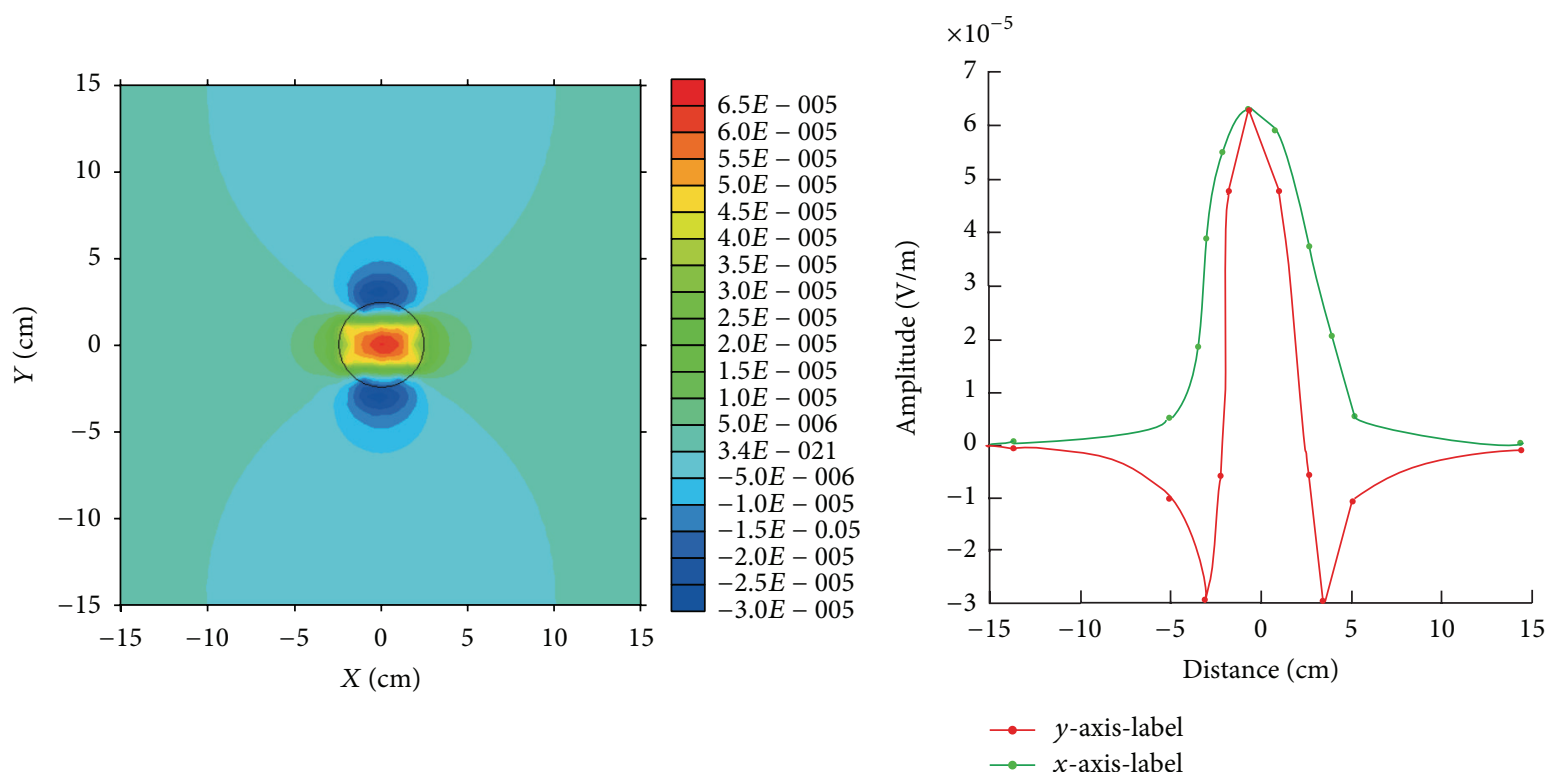

(a)
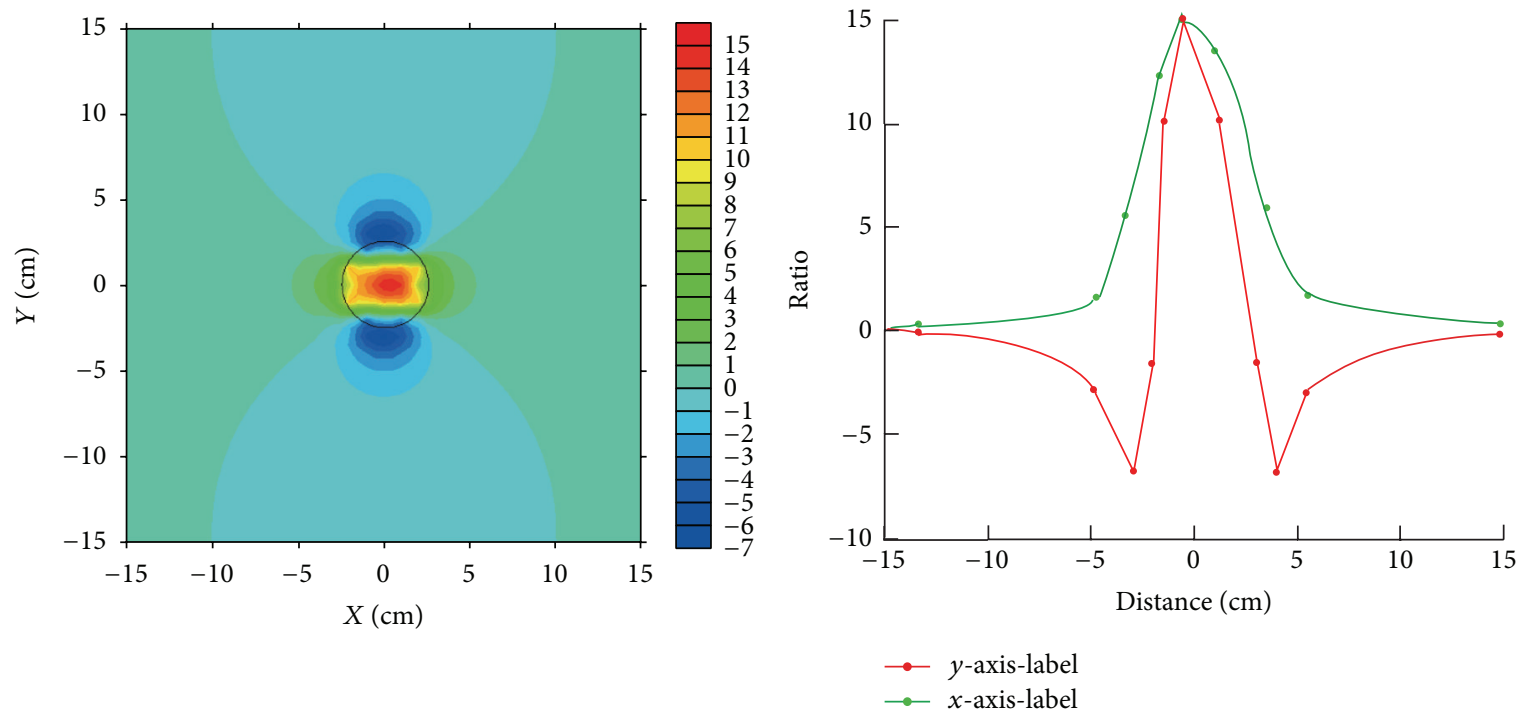

(c)

(d)

Figure 2: (a) Distribution of the $E_{y}$ component of the anomalous field on the plane $z=1000 \mathrm{~cm}$. (b) Distribution of the $E_{y}$ component of the anomalous field along the $x$-and $y$-axis-label on the plane $z=1000 \mathrm{~cm}$. (c) Ratio of the $E_{y}$ component's anomalous field to the background field on the plane $z=1000 \mathrm{~cm}$. (d) Ratio of the $E_{y}$ component's anomalous field to the background field along the $x$-and $y$-axis-label on the plane $z=1000 \mathrm{~cm}$.

The influence of the XCP probe was minor and would eventually disappear when the observation points were located far enough from the probe. Based on the boundary conditions that current density was continuous in the normal direction at the outer and inner sides of the XCP probe, we can obtain the following equations:

$$
\begin{aligned}
j_{1 n} & =j_{2 n}, \\
\frac{E_{1 n}}{\rho_{1}} & =\frac{E_{2 n}}{\rho_{2}} .
\end{aligned}
$$

The terms $j_{1 n}$ and $j_{2 n}$ are the scattered currents inside and outside of the XCP probe, respectively; $E_{1 n}$ and $E_{2 n}$ are the electric field intensities inside and outside of the XCP probe, respectively; and $\rho_{1}$ and $\rho_{2}$ are the densities inside and outside of the XCP probe, respectively. Because the resistivity of the XCP probe was higher than that of seawater, the electric field within the probe was much greater than the external anomalous electric field.

The distribution characteristics of the $E_{y}$ component's anomalous field and current-induced electric field, known as background field ratios, are shown in Figures 2(c) and 2(d), 


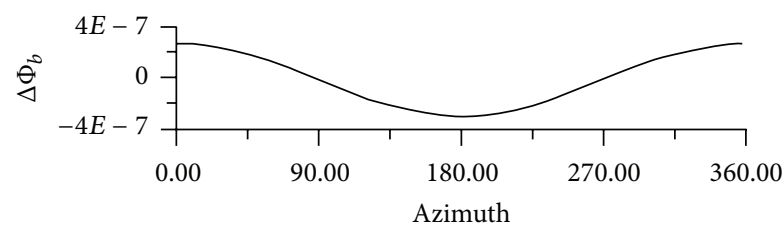

(a)

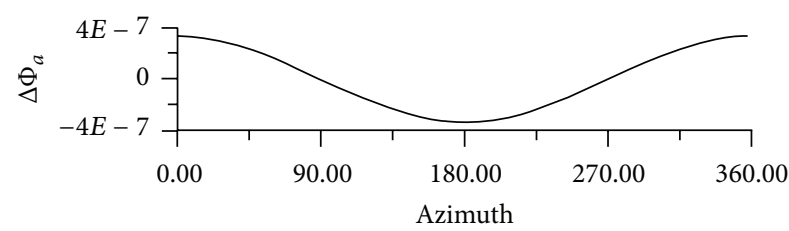

(b)

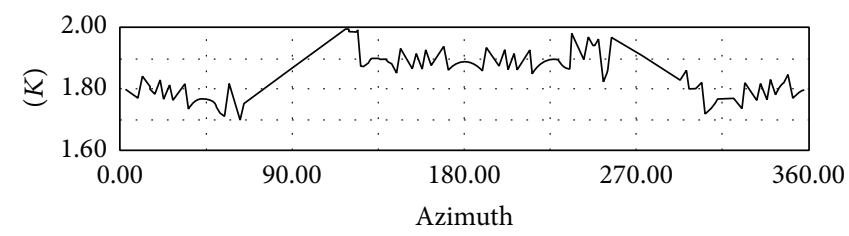

(c)

FIGURE 3: Changes in electric potential difference and voltage enhancement factor as a function of the azimuth. (a) Electric potential difference caused by the background field. (b) Electric potential difference caused by the anomalous field. (c) $K$ as a function of the azimuth.

respectively. The distribution characteristics of Figure 2(c) are basically similar to those of Figure 2(a). At the upper and lower sides of the XCP probe, the smallest anomalous field was approximately -6 times the size of the background field. There are positive anomalous characteristics on the left and right sides of the probe, where the ratio of the anomalous field to the background field is 7 .

The simulation results below indicated that the amplitude of the electric field on the observation plane was significantly influenced by the probe. The distribution was symmetrical along the $y$-axis, whereas the ratio of the anomalous field to the background field varied with respect to the location of the observation points. In this situation, in-depth computational analyses must be carried out in order to determine whether placing the probe in seawater would influence the measured voltage.

\subsubsection{Calculating the Probe's Influence on Measured Voltages.} Electric field distributions obtained from forward modelling were required for the analysis of voltage changes. These were then used to calculate the electric potential difference between the two electrodes [20]. The electric potential difference was actually obtained by integrating the electric field's intensity vectors measured along a particular path between one electrode and the other. (The two electrodes were located $5 \mathrm{~cm}$ apart at both ends of the probe's minor axis and constituted two observation points on a straight line.)

The solid circular line represents the cross-section passing through the centre of the instrument, whereas the dots represent the outer side of the instrument. The outer diameter of the instrument was $5 \mathrm{~cm}$, and the major axis of the ellipsoid was $52 \mathrm{~cm}$.

When the electric field vectors $\vec{E}_{b}$ and $\vec{E}_{a}$ were separately applied to (25) below, the electric potential differences caused by the background and anomalous fields between the two electrodes were obtained as $\Delta \Phi_{b}$ and $\Delta \Phi_{a}$, respectively, after integration. $\Delta \Phi_{b}$ corresponds to the electric potential difference between the two electrodes when the probe was not inserted, whereas $\Delta \Phi_{b}+\Delta \Phi_{a}$ corresponds to the electric potential difference between the electrodes after insertion of the probe. This can be used to calculate the increase of $K$ between the original electric potential difference of the two electrodes and that after insertion of the probe, as shown in (26).

$$
\begin{aligned}
\Delta \Phi & =\int \vec{E} \cdot \vec{l} d l, \\
K & =\frac{\Delta \Phi_{b}+\Delta \Phi_{a}}{\Delta \Phi_{b}} .
\end{aligned}
$$

By changing the angle between the two electrodes and the external electric field, we were able to observe the way in which $K$ varied for various azimuths. The calculation results when the diameter of the probe's minor axis was $5 \mathrm{~cm}$ are shown in Figure 3. It can be seen that the electric potential difference caused by the background and anomalous fields exhibited similar patterns as the azimuth varied. The calculated $K$ values indicated that measurements of the electric field were significantly influenced after insertion of the probe. The average of the various $K$ values was calculated, which yielded a value of 1.85 in this example.

3.1.4. Variations in Diameter of Probe's Minor Axis and the Influence on $K$. The calculated values of $K$ are shown in Table 1 . It can be seen that changes in the value of $K$ were bigger when the minor axis was $5 \mathrm{~cm}$ long; the values of $K$ were relatively lower for lengths of 20 and $52 \mathrm{~cm}$. It turns that all of the conditions are in the range of the errors permitted.

3.1.5. Variations in Signal Frequency of Electric Dipole Source and the Influence on $K$. Numerical simulations of the electric field were performed using the model in which the length of the probe's minor axis was $5 \mathrm{~cm}$; the signal frequencies of the electric dipole source were then varied. The frequencies used during the simulations were $0,0.0001$, and $1 \mathrm{~Hz}$. Data concerning the electric field obtained from the simulations were then used to calculate the corresponding changes in the value of $K$ as a function of the azimuth; the results are shown in Figure 4. The values of $K$ were relatively large (average: 1.85) when the frequency of the transmission signal was $0 \mathrm{~Hz}$. 
TABLE 1: Average value of $K$ under different conditions.

\begin{tabular}{lcccccc}
\hline Length of minor axis $(\mathrm{cm})$ & 2 & 4 & 5 & 6 & 10 & 20 \\
Average value of $K$ & 1.74 & 1.73 & 1.85 & 1.75 & 1.80 & 1.68 \\
Error coefficient & $\pm 6.5 \%$ & $\pm 6.7 \%$ & $\pm 1.5 \%$ & $\pm 6.5 \%$ & $\pm 2 \%$ & $\pm 7.2 \%$ \\
\hline
\end{tabular}

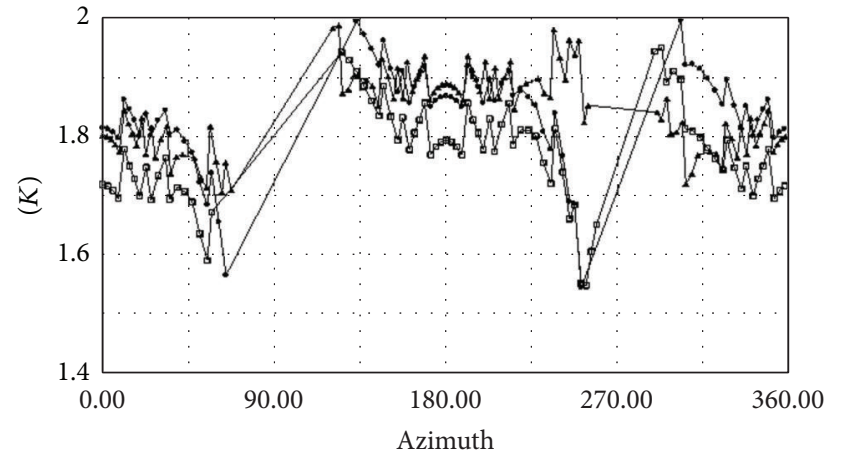

Frequency

$\simeq 0 \mathrm{~Hz}$

$\rightarrow 0.0001 \mathrm{~Hz}$

$\square 1 \mathrm{~Hz}$

FIGURE 4: Changes in voltage increase rate as a function of the azimuth for different signal frequencies.

These decreased slightly (average: 1.84) at the frequency of $0.0001 \mathrm{~Hz}$ but were relatively small (average: 1.77 ) at the frequency of $1 \mathrm{~Hz}$. These results indicate that the voltage increase rate exhibited a decreasing trend as the frequency increased.

3.2. Physical Simulations of Probe's Influence on Electric Field of Ocean Currents. In order to further understand the trends discussed above and to confirm the probe's influence on IEF measurements, physical simulations were carried out.

3.2.1. Test Environment. The electric ionization currents of industry create too much interference when a regular water tank is used to measure electric fields. Hence, physical simulations for this study were conducted indoors using a large plastic container. In order to simulate a genuine seawater environment, the conductivity of seawater was adjusted to be $3.3 \mathrm{~S} / \mathrm{m}$. The main used instruments and equipment consisted of a signal recovery 7265 DSP lock-in amplifier, which operated over a frequency range of $1 \mathrm{mHz}$ to $250 \mathrm{kHz}$; model 7265 offers full-scale voltage sensitivities down to $2 \mathrm{nV}$ and current sensitivities to $2 \mathrm{fA}$. We also used Matrix MPS-3003L-3 (voltage display precision: three and a half $\mathrm{A} / \mathrm{D}$ conversion digital display, $\pm 0.5 \%+2$ words; current display precision: three and a half $\mathrm{A} / \mathrm{D}$ conversion digital display, $\pm 1 \%+2$ words). Furthermore, we used Tektronix TDS 2002 (vertical resolution: 8 bits; vertical sensitivity: $2 \mathrm{MV}$ to $5 \mathrm{~V} /$ div; DC vertical precision: $\pm 3 \%$ ), Agilent $34420 \mathrm{~A}$ (display resolution: $7^{1 / 2}$; sensitivity: $100 \mathrm{pV} / \mathrm{n} \Omega$ ), Hewlett Packard 33120A (accuracy at $1 \mathrm{kHz}: \pm 1 \%$ of specified output), Victor VC9801A + (DC voltage: $\pm(0.5 \%+3)$; AC voltage: $\pm(0.8 \%+5)$; DC current: $\pm(0.8 \%+10)$; AC current: $\pm(1.0 \%+15))$. A personal computer (PC), two copper plates that supplied electricity, two small $\mathrm{Ag} \mid \mathrm{AgCl}$ nonpolarizable electrodes, and a solid highresistance cylinder (outer diameter: $5 \mathrm{~cm}$ ) were also used.

The underwater measuring environment dictates that the signal of the ocean currents' electric field must undergo a transmission process from a liquid to a solid medium. Normal nonpolarizable electrodes create electrochemical noise when the electrodes come into mutual contact, which is extremely unfavourable when observing the weak IEF signals within ocean currents [21]. For this reason, the first step in carrying out physical simulations was to search for an electrode material with a small yet steady polarization potential when placed in a marine environment. Many past experiments have shown that when silver and silver chloride in powder form are mixed according to a specific formula and then made into electrodes using metallurgical processes, the latter exhibit good electrochemical properties when placed in seawater [22]. This could be explained through the conductive mechanism of $\mathrm{Ag} \mid \mathrm{AgCl}$ itself. First, compared to other electrode materials, it is easier to refine Ag to its pure state under laboratory conditions, thereby eliminating any "battery effect" [23] caused by impurities (one of the noise sources). Pure Ag also has better electrochemical stability in an environment with a generally constant temperature. (The temperature of seawater can be almost constant across an extremely short period of time and within a specific area.) Second, Clions are the material carriers of seawater conductivity. After AgCl comes into contact with seawater, the same chemical composition is also the main carrier that conducts electricity at the contact surface between the solid and liquid phases [24].

3.2.2. Test Contents. The tests were carried out as the copper plates were charged and discharged and as the conditions for supplying electricity and making measurements were varied; the electric field was stabilized during these situations. Under direct current conditions, the voltage was measured before and after the high-resistance cylinder was placed in the brine. Under alternating current conditions, the voltage and supply current were measured before and after the high-resistance cylinder was placed in the brine.

3.2.3. Tests Using Electric Field with Direct Current. The voltage source Matrix supplied $2 \mathrm{~V}$ of constant voltage to the copper plates in the simulation water tank; the copper plates were connected to the PC and Agilent 34420A. This process involved first placing the high-resistance cylinder into the water for $30 \mathrm{~min}$ of data collection, followed by another 30 min of data collection after removal of the cylinder. Five groups of data were measured during the tests. The measurements for Group 1, as shown in Figures 5(a) and 5(b), indicate that under both test conditions and with a stable power supply, the collected voltage signals would stabilize after a short 


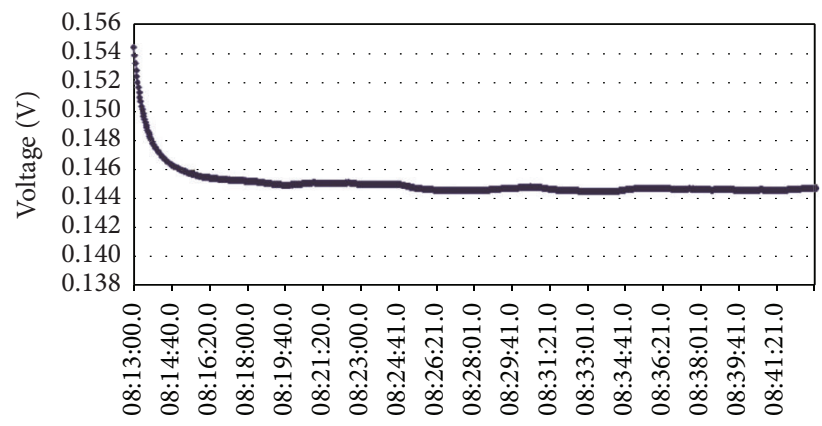

(a) Test data for Group 1 (with high-resistance cylinder)

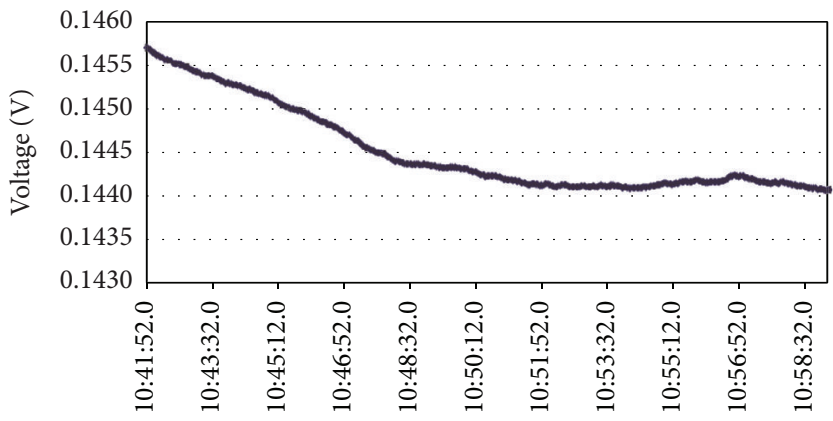

(c) Test data for Group 2 (with high-resistance cylinder)

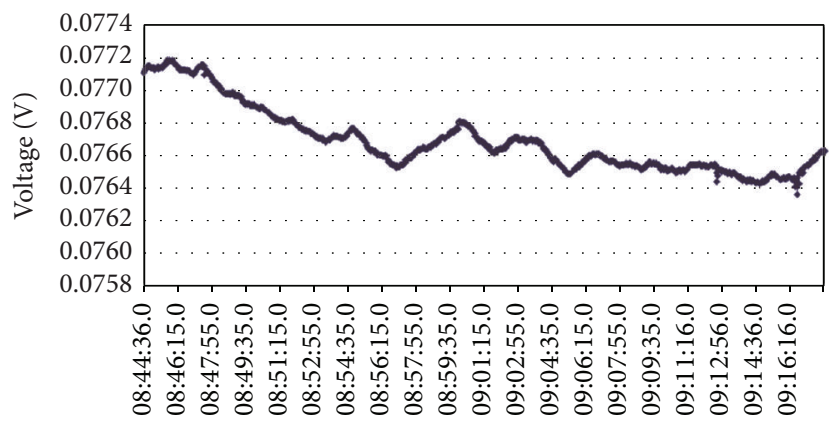

(b) Test data for Group 1 (without high-resistance cylinder)

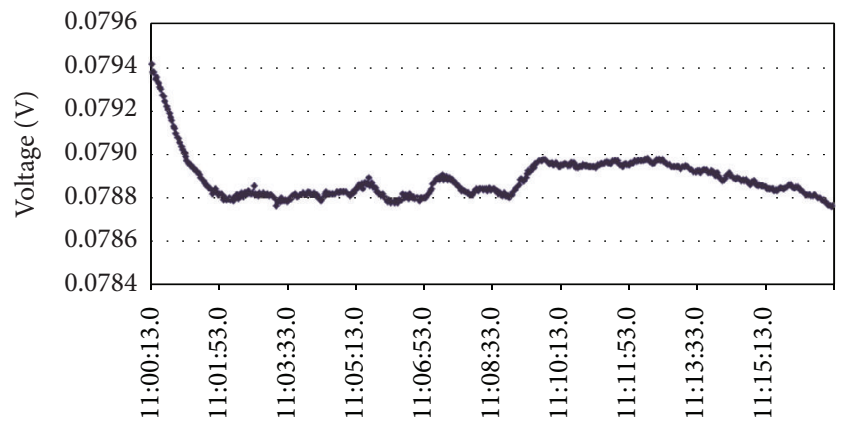

(d) Test data for Group 2 (without high-resistance cylinder)

FIGURE 5: (a) Measured voltage between electrodes for Group 1 after being supplied with electricity (with high-resistance cylinder). (b) Measured voltage between electrodes for Group 1 after being supplied with electricity (without high-resistance cylinder). (c) Measured voltage between electrodes for Group 2 after being supplied with electricity (with high-resistance cylinder). (d) Measured voltage between electrodes for Group 2 after being supplied with electricity (without high-resistance cylinder).

TABLE 2: Test data for influence of high-resistance cylinder on electric field.

\begin{tabular}{|c|c|c|c|c|}
\hline \multirow{2}{*}{ Five groups of data } & \multicolumn{2}{|c|}{ High-resistance cylinder } & \multirow{2}{*}{ K } & \multirow{2}{*}{ Average value of $K$} \\
\hline & With $(V)$ & Without $(V)$ & & \\
\hline Group 1 & 0.146640334 & 0.077900667 & 1.882 & \\
\hline Group 2 & 0.144190749 & 0.078882746 & 1.828 & \\
\hline Group 3 & 0.145283745 & 0.079246566 & 1.833 & 1.826 \\
\hline Group 4 & 0.143979815 & 0.078936453 & 1.824 & \\
\hline Group 5 & 0.142412165 & 0.077983919 & 1.826 & \\
\hline
\end{tabular}

period of 2-3 min. During the measurement process, the supply current signals were also recorded at the appropriate intervals. The purpose of this was to normalize the electric currents, thereby eliminating the influence of current changes on the measurement data. The measured voltages under the two test conditions were approximately 78 and $145 \mathrm{mV}$, respectively. The maximum relative error caused by the potential difference between the nonpolarizable electrodes within 30 min did not exceed $1 \%$. Thus, the influence of the potential difference between electrodes on the measurements was ignored during subsequent calculations and analyses.

The measurements for Group 2 are shown in Figures 5(c) and $5(\mathrm{~d})$. For each group of data, the average of two voltages was used to calculate the ratio of the electrode voltage with the high-resistance cylinder to that without the cylinder (i.e., the voltage increase rate $K$ ). Data for the five sets of measurements are shown in Table 2.
3.2.4. Tests Using Electric Field with Alternating Current. The 7265 DSP lock-in amplifier by signal recovery was used for these tests. The alternating signals from the lock-in amplifier were loaded onto the copper plates of the water tank. The size of the signal was adjusted to control the strength of the signal received by the electric field sensor. The test data are shown in Table 3.

\section{Conclusions}

This study investigated the influence that the probes used for XCP detection have on the IEF of ocean currents. Computerbased numerical simulations were used as the basis for physical tests that were made in simulated marine environments. Based on the assumption that both simulations were carried out under similar conditions, the conclusions from the theoretical analysis are as follows. The amplitude of the 
TABLE 3: Test data for influence of high-resistance cylinder on $15 \mathrm{~Hz}$ alternating electric field (dilute brine).

\begin{tabular}{|c|c|c|c|c|c|c|c|}
\hline \multirow{3}{*}{ Group number } & \multicolumn{7}{|c|}{ High-resistance cylinder } \\
\hline & \multirow[t]{2}{*}{ Output rms of lock-in amplifier } & \multicolumn{2}{|c|}{ With } & \multicolumn{2}{|c|}{ Without } & \multirow[t]{2}{*}{$K$} & \multirow[t]{2}{*}{ Average value of $K$} \\
\hline & & $\mathrm{CP}$ & LA & $\mathrm{CP}$ & LA & & \\
\hline Group 1 & $2 \mathrm{~V}$ & $339 \mathrm{mV}$ & $73.92 \mathrm{mV}$ & $339 \mathrm{mV}$ & $49.0 \mathrm{mV}$ & 1.509 & \multirow{8}{*}{1.502} \\
\hline Group 2 & $1 \mathrm{~V}$ & $163 \mathrm{mV}$ & $38.03 \mathrm{mV}$ & $163 \mathrm{mV}$ & $25.33 \mathrm{mV}$ & 1.501 & \\
\hline Group 3 & $0.1 \mathrm{~V}$ & $16.5 \mathrm{mV}$ & $3.80 \mathrm{mV}$ & $16.5 \mathrm{mV}$ & $2.53 \mathrm{mV}$ & 1.502 & \\
\hline Group 4 & $10 \mathrm{mV}$ & - & $381 \mu \mathrm{V}$ & - & $255.8 \mu \mathrm{V}$ & 1.489 & \\
\hline Group 5 & $1 \mathrm{mV}$ & - & $38.17 \mu \mathrm{V}$ & - & $25.25 \mu \mathrm{V}$ & 1.512 & \\
\hline Group 6 & $100 \mu \mathrm{V}$ & - & $3.70 \mu \mathrm{V}$ & - & $2.450 \mu \mathrm{V}$ & 1.510 & \\
\hline Group 7 & $10 \mu \mathrm{V}$ & - & $361 \mathrm{nV}$ & - & $241 \mathrm{nV}$ & 1.498 & \\
\hline Group 8 & $2 \mu \mathrm{V}$ & - & $74 \mathrm{nV}$ & - & $49.5 \mathrm{nV}$ & 1.495 & \\
\hline
\end{tabular}

$\mathrm{CP}$ : voltage rms of copper plate supplying electricity.

LA: measured rms of lock-in amplifier.

IEF being measured was significantly influenced by the probe. For a probe with a minor axis whose diameter was $5 \mathrm{~cm}$, the maximum voltage-enhancement factor based on theoretical calculations was 1.85 . Using the data from the simulated physical tests, the coefficient of influence of a probe with similar dimensions on an electric field was 1.826. Ultimately, the conclusions for both types of simulation were basically similar. These results verified the influence that the probes had on the IEF of ocean currents and illustrated that electric fields could be strengthened through the rational design of probe dimensions, thereby facilitating the use of the XCP in monitoring marine environments.

\section{Competing Interests}

All of the contributing authors of this article declare that there is no conflict of interests regarding the publication of this paper.

\section{Acknowledgments}

This work was supported by the Natural Science Foundation of China (nos. 41574131 and 41204135), the National "863" Program of China (nos. 2012AA061102 and 2012AA09A20102), and the Fundamental Research Funds for the Central Universities of China (no. 2652015213).

\section{References}

[1] T. B. Sanford, R. G. Drever, and J. H. Dunlap, "A velocity profiler based on the principles of geomagnetic induction," Deep-Sea Research, vol. 25, pp. 183-210, 1978.

[2] T. B. Sanford, "Motionally induced electric and magnetic fields in the sea," Journal of Geophysical Research, vol. 76, no. 15, pp. 3476-3492, 1971.

[3] J. H. Dunlap, R. G. Drever, and T. B. Sanford, "Experience with an expendable temperature and velocity profiler," in Proceedings of the OCEANS, pp. 372-376, September 1981.

[4] T. B. Sanford, "Velocity profiling: some expectations and assurances," in Proceedings of the IEEE 2nd Working Conference on Current Measurement, M. Dursi and W. Woodward, Eds., pp. 101-112, New York, NY, USA, 1982.
[5] Z. B. Szuts, "Using motionally-induced electric signals to indirectly measure ocean velocity: Instrumental and theoretical developments," Progress in Oceanography, vol. 96, no. 1, pp. 108127, 2012.

[6] N. Liu and H.-K. He, "Study on the theory of expendable current profiler measurement," Ocean Technology, vol. 1, pp. 8-11, 2010.

[7] Q.-S. Zhang, M. Deng, N. Liu, Y.-G. Kong, and S.-L. Guan, "Development of the expendable current profiler," Chinese Journal of Geophysics, vol. 56, no. 11, pp. 3699-3707, 2013 (Chinese).

[8] Q. S. Zhang, M. Deng, and Q. Wang, "Dynamic data transmission technique for expendable current profiler," Advanced Materials Research, vol. 220, pp. 436-440, 2011.

[9] N. Liu, Y. J. Li, and G. W. Zhu, "A kind of fast expendable current profiler measure production," The Journal of Ocean Technology, vol. 26, pp. 27-31, 2007 (Chinese).

[10] W.-Y. Chen, R. Zhang, N. Liu, and M.-M. Zhang, "Numerical study on the influence of rotating to the movement characteristics of XCP probe," Ocean Technology, vol. 30, pp. 61-63, 2011 (Chinese).

[11] Q. Wang, D. Zhang, and J. Sun, "Preliminary probe into sustainable development and application of ocean resources," China Population, Resources and Environment, vol. 2, pp. 26-28, 2000.

[12] M. Deng, W. Wei, H. Tan, S. Jin, and J. Deng, "Difficulties in the marine magnetotelluric signal acquisition," Geoscience, vol. 16, no. 1, pp. 94-99, 2002.

[13] P. E. Wannamaker, G. W. Hohmann, and W. A. Sanfilipo, "Electromagnetic modeling of three-dimensional bodies in layered earths using integral equations," Geophysics, vol. 49, no. 1, pp. 60-74, 1984.

[14] P. E. Wannamaker, "Advances in three-dimensional magnetotelluric modeling using integral equations," Geophysics, vol. 56, no. 11, pp. 1716-1728, 1991.

[15] G. W. Hohmann, “Three-dimensional induced polarization and electromagnetic modeling," Geophysics, vol. 40, no. 2, pp. 309324, 1975.

[16] Z.-G. Wang, Z.-X. He, and W.-B. Wei, "Research on 3D modeling of borehole vertical bipole using body integral equation," Progress in Geophysics, vol. 22, no. 6, pp. 1802-1808, 2007.

[17] H. Zhang, T.-L. Li, and R.-X. Dong, "3D Electromagnetic inversion by volume integral equation method based on current dipole source," Journal of Jilin University (Earth Science Edition), vol. 2, pp. 284-288, 2006. 
[18] H. Zhang, T.-L. Li, and R.-X. Dong, "Modeling 3-D electromagnetic responses of the electric dipole using volume integral equation method," Progress in Geophysics, vol. 2, pp. 386-390, 2006.

[19] Z.-L. Zhang, W.-B. Wei, B.-H. Liu, M. Deng, and S. Jin, "Theoretical calculation of electromagnetic field generated by ocean waves," Acta Oceanologica Sinica, vol. 1, pp. 42-46, 2008.

[20] J. C. Larsen and T. B. Sanford, "Florida current volume transports from voltage measurements," Science, vol. 227, no. 4684, pp. 302-304, 1985.

[21] D. Ming, W. Wenbo, D. Jingwu, and T. Handong, "Lab simulation tests for the coming submarine magnetotelluric survey," Journal of Shantou University, vol. 17, pp. 20-71, 2002.

[22] Y.-G. Wei, Q. Cao, Y. Huang, and Y. Wang, "Preparation and properties of $\mathrm{Ag} / \mathrm{AgCl}$ electrode with low noise of marine electric field sensor," Journal of Synthetic Crystals, supplement 1, pp. 394-398, 2009.

[23] M. Deng, W.-B. Wei, P. Yu, Z.-S. Chen, J.-W. Deng, and L.$\mathrm{X}$. Li, "The marine experiments of seafloor magnetotelluric prospecting," Geoscience, vol. 16, pp. 443-447, 2002.

[24] F.-L. Huang, Q.-X. Cao, and Y.-G. Wei, "Preparation and electrochemical performance of $\mathrm{Ag} / \mathrm{AgCl}$ electrodes," Electronic Science and Technology, vol. 6, pp. 29-31, 2010. 


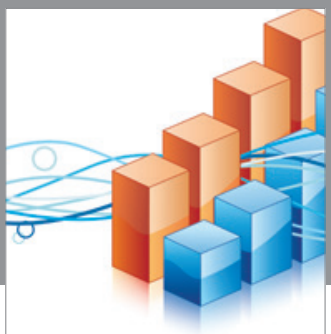

Advances in

Operations Research

vatem alat4

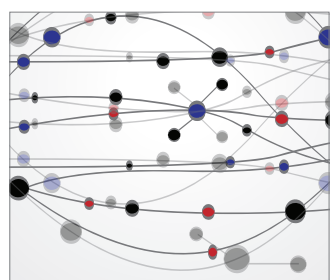

\section{The Scientific} World Journal
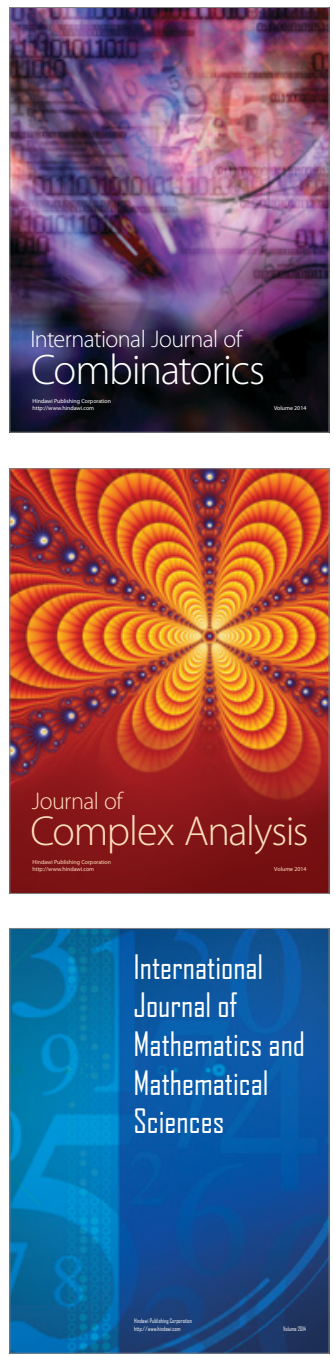
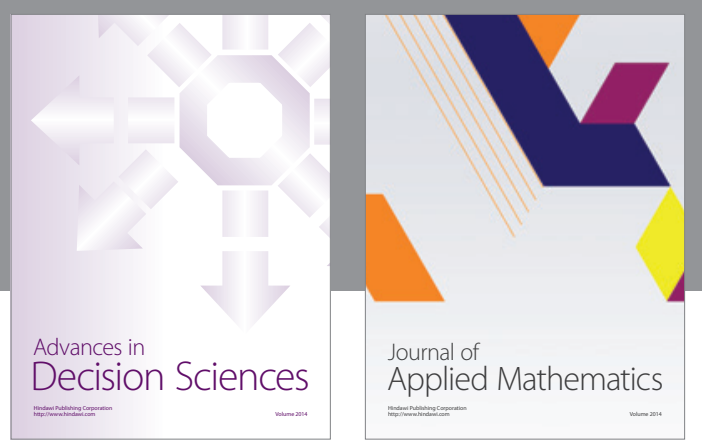

Algebra

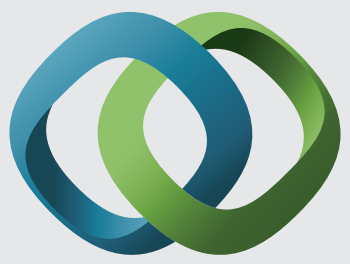

\section{Hindawi}

Submit your manuscripts at

http://www.hindawi.com
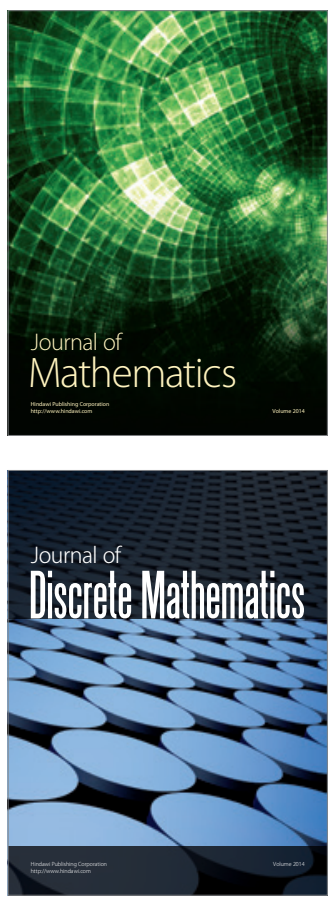

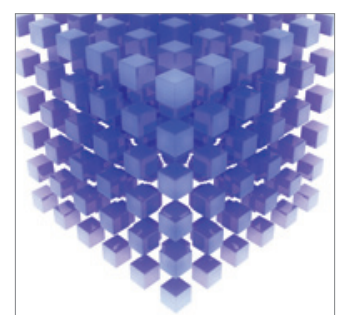

Mathematical Problems in Engineering
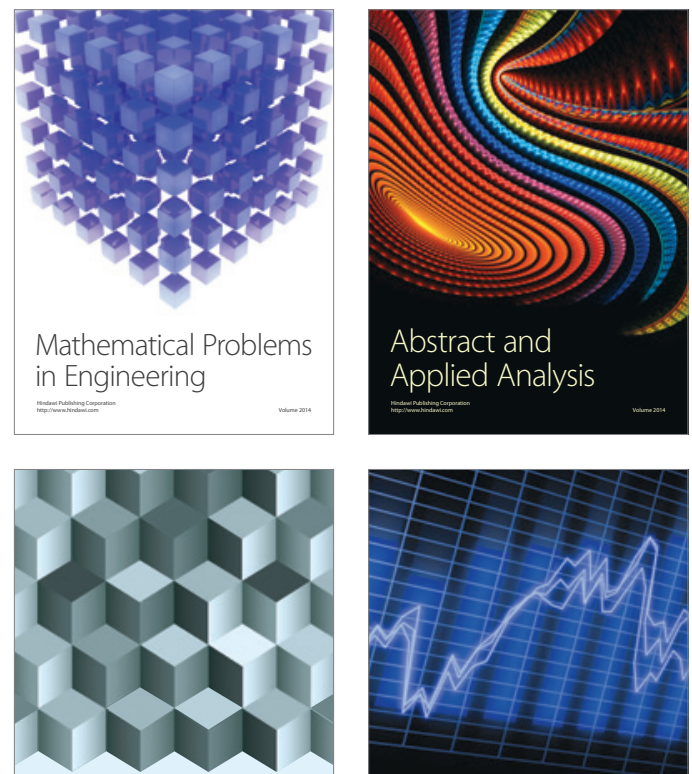

Journal of

Function Spaces

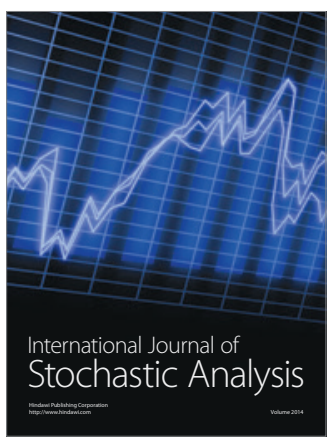

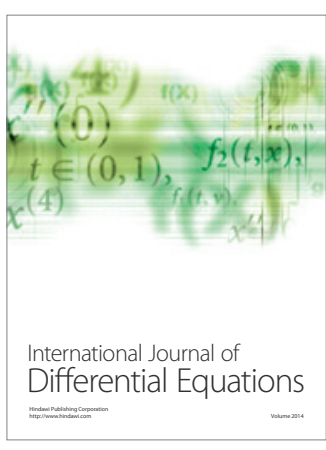
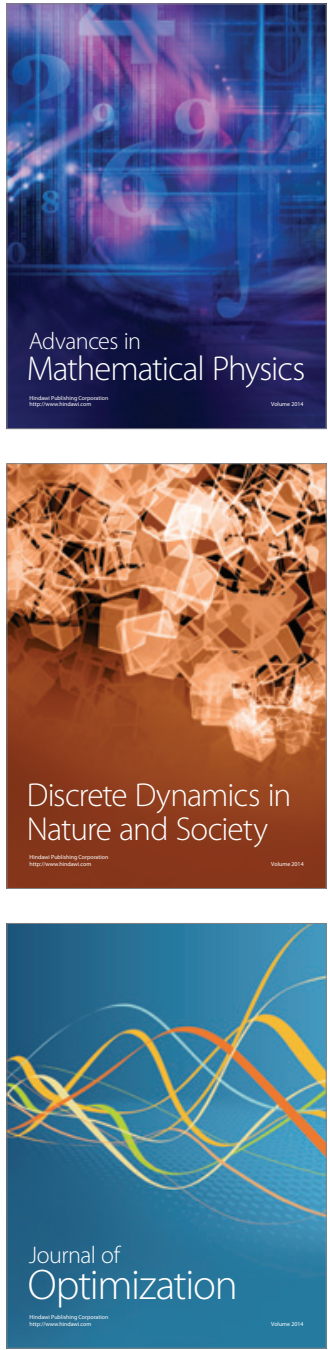